\title{
RESOLUTION ENHANCEMENT ALGORITHM FOR SPACEBORN SAR BASED ON HANNING FUNCTION WEIGHTED SIDELOBE SUPPRESSION
}

\author{
Caiping $\mathrm{Li}^{1}$, Xiaoming Zhou ${ }^{1}$, Dejin Tang ${ }^{2}$, Zhuhua Zhu ${ }^{2, *}$ \\ ${ }^{1}$ Beijing Institute of Remote Sensing Information,100192, Haidian District ,Beijing,China-zxm2913@163.com \\ ${ }^{2}$ National Geomatics Center of China, 100830,28\#Lianhuachi road, BeiJing, China-tangdejin@ngcc.cn
}

\section{Commission III, WG III/3}

KEY WORDS: SAR image, Sidelobe suppression, Hunning function

\begin{abstract}
:
Resolution and sidelobe are mutual restrict for SAR image. Usually sidelobe suppression is based on resolution reduction. This paper provide a method for resolution enchancement using sidelobe opposition speciality of hanning window and SAR image. The method can keep high resolution on the condition of sidelobe suppression. Compare to traditional method, this method can enchance $50 \%$ resolution when sidelobe is $-30 \mathrm{~dB}$.
\end{abstract}

\section{INTRODUCTION}

Resolution and sidelobe level are two key indicators that reflect the quality of SAR images. Due to the limitation of the SAR imaging mechanism, to achieve the highest resolution of the satellite system, the peak sidelobe ratio of the ground SAR data is only $-13 \mathrm{~dB}$. However, the peak sidelobe ratio of $-13 \mathrm{~dB}$ interferes with the surrounding target, causing the target to be difficult to identify. Usually, the sidelobe suppression is carried out on the ground. The peak sidelobe ratio of foreign civil radar satellites is suppressed to $-20 \mathrm{~dB}$, and the peak sidelobe ratio of military radar satellites is suppressed to -30dB. SAR in Chinese academic circles often uses the weighted method for sidelobe suppression, but the SAR image weighting method, image resolution and peak sidelobe ratio is a restriction of the index, to sacrifice a part of sidelobe suppression required to obtain high resolution, the resolution must tolerate high sidelobe level. The sidelobe level is usually suppressed to $-20 \mathrm{~dB}$, and the resolution will lose about 20\%. Document [3]-[4] has studied the reconstruction method of SAR imaging based on compressed sensing, which can achieve better sidelobe suppression effect, but this method is only suitable for imaging sparse targets such as ocean background. This paper aims to study a new SAR image enhancement technology, while maintaining the resolution, effectively reduce the sidelobe level, thereby improving the image quality, improve the capability of target identification, image processing of any background of the target methods can get good effect. Or, in the condition of sidelobe suppression, the ideal resolution of the satellite system can be achieved.

\section{SAR IAMGE RESOLUTION RATION}

Ideally, the ground range resolution of the SAR image $\delta_{g}$ is expressed as:

$$
\delta_{g}=0.886 \frac{c}{2 B \sin \theta_{1}}
$$

In which $B$ represents the bandwidth of the transmitted signal, and the $\mathrm{C}$ is the speed of light, $\theta_{1}$ which is the angle of incidence.

The azimuthal resolution direction is expressed as:

$$
\delta_{a z}=k_{\delta} k_{W}\left\{0.886 \frac{\lambda}{2 \theta_{0}}\right\}
$$

In which $\lambda$ is the working wavelength , and $\theta_{0}$ is the angle of the radar system by passing the target flight.

The actual imaging, as a result of the radar system, the Doppler frequency rate estimation, wave propagation and imaging processing errors caused by poor image resolution system, $K_{\delta}$ is the definition of main lobe broadening coefficients of these error, it directly reflects the effect of radar system, satellite platform and image processing algorithms of the resolution. On the other hand, in image processing, the necessary weighting to reduce the sidelobe is to sacrifice the resolution, resulting in the image main lobe broadening and image resolution becoming worse. $K_{W}$ is the main lobe broadening coefficient defined as weighted by imaging processing. Considering the effects of these errors, the ground range and azimuthal resolution are respectively expressed as:

$$
\begin{gathered}
\delta_{g}=k_{\delta} k_{w}\left\{0.886 \frac{c}{2 B \sin \theta_{1}}\right\} \\
\delta_{a z}=k_{\delta} k_{w}\left\{\frac{\lambda}{2 \theta_{0}}\right\}
\end{gathered}
$$

Formula (3) and formula (4) parenthesis parameters are determined by imaging principle and system parameters. $k_{\delta}$ is the main lobe broadening coefficient which can be controlled in a relatively small range through system design, 
system implementation, imaging algorithm optimization and error compensation, but it can not be completely eliminated.

$K_{W}$ is the main lobe broadening coefficient that is caused by the necessary weighting in order to reduce the sidelobe in imaging processing. When the sidelobe is usually suppressed from $13 \mathrm{~dB}$ to $-20 \mathrm{~dB}$, it is about 1.2 , that is, the resolution is about $20 \% . K_{W}$ can be eliminated through the SAR image resolution enhancement processing technology basically.

SAR image resolution enhancement processing technology is of great application value. In order to get high resolution, satellite system often has to pay very high development cost, increase the difficulty and risk of developing the system, and even sacrifice other image quality indicators, such as imaging bandwidth. If a high resolution can be obtained through the imaging process and does not affect the other image quality indicators, it is a very ideal result. The image enhancement method proposed in this paper can suppress the sidelobe level to

$-30 \mathrm{~dB}$ and $k_{W}$ is approximate to 1 , which means that the image resolution is basically free from loss.

\section{IMAGE ENHANCEMENT PROCESSING}

For SAR images, in order to lower the sidelobe without loss of resolution, the side lobe must be lowered and the width of the main discrimination can not be broadened. Therefore, to enhance the method proposed in the paper, the main use of sidelobe weighting function spectrum is consistent with a single complex image signal spectrum sidelobe width, but the amplitude characteristics of the opposite to get the signal value, in the width of the main lobe, in order to guarantee the same and the resolution of the signal; Outside the width of the main lobe, due to the original signal and a weighted function amplitude of positive and negative offset each other, instead, is outside the main lobe width value is zero, in order to guarantee a higher ratio of peak sidelobe.

It should be emphasized that the enhancement processing method is to make use of the high-precision phase characteristics of SAR images. Therefore, phase error correction is necessary in the enhancement process. In addition, the azimuth and distance direction are all processed under unweighted conditions, and the weighted image processing must be de weighted.

\subsection{De weighted treatment}

Generally, there are two methods of de-weighting, namely, direct de-weighting and fitting modulation.

When the weighted function is known, the direct de weighting method is used, because the weighting is completed in the frequency domain, so the de weighting also needs to be carried out in the frequency domain.

$Y(k, 1)$ is a representation of the weighted SAR image in the azimuth and distance frequency domain, $W$ is to reduce the weighted function used by the sidelobe, and the frequency domain data after the de-weighting $X(k, l)$ are as follows:

$$
X(k, l)=Y(k, 1) / W
$$

When the weighting function is uncertain, we can use the fitting modulation method to fit the spectrum amplitude, and then remove the fitting value in the spectrum, so that the spectrum amplitude approaches to a constant. The common fitting methods are linear fitting, nearest point fitting, low - pass fitting and polynomial fitting.

\subsection{Phase error correction}

The phase error gradient estimation method is as follows:FFT for time domain signals.

$$
X(k)=|X(k)| \exp \left(j\left[\phi_{\varepsilon}(k)+\theta(k)\right]\right)
$$

$\mathrm{K}$ is the azimuthal coordinate in the frequency domain, $\phi_{\varepsilon}(k)$ is the azimuth phase error in the frequency domain, $\theta(k)$ is the residual phase term. Error phase gradient.

$$
\Delta \phi_{\varepsilon}(k)=\angle\left\{X(k) X^{*}(k-1)\right\}
$$

Phase error gradient summation:

$$
\phi_{\varepsilon}(k)=\sum_{m=1}^{k} \Delta \phi_{\varepsilon}(m)
$$

The phase error curve $\phi_{\varepsilon}(k)$ is obtained, and the fixed phase and linear term are removed. The $\mathrm{X}(\mathrm{k})$ compensates the phase error $\phi_{\varepsilon}(k)$ to obtain the spectrum data after the phase error compensation.

\subsection{Sidelobe inhibition}

$X(k, l)$ is the set to the original SAR image in the weighted representation of azimuth and distance in frequency domain, hanning weighting function is denoted by:

$$
{ }_{W}(n)=1-\cos \left[\frac{2 \pi n}{N}\right]
$$

Hanning weighting function for frequency domain representation:

$$
W(k)=\operatorname{FFT}\{W(n)\}
$$

The time domain signal $X_{1}(n, m)$ and the original domain signal $X_{2}(n, m)$ by hanning weighted are respectively:

$$
\begin{aligned}
X_{1}(n, m) & =\operatorname{IFFT}\{W(k) X(k, l)\} \\
& =X_{1}(n, m)+j X I_{1}(n, m) \\
X_{2}(n, m) & =\operatorname{IFFT}\{X(k, l)\} \\
& =X_{2}(n, m)+j X I_{2}(n, m)
\end{aligned}
$$

The real part and the imaginary part of $X_{1}(n, m)$ and $X_{2}(n, m)$ is compared respectively, and if $X R_{1}(n, m)$ and $X R_{2}(n, m)$ are with the different number, then it is taken:

$$
Y_{R}(n, m)=0
$$

If $X R_{1}(n, m)$ and $X R_{2}(n, m)$ are same, then it is taken:

$Y_{R}(n, m)=\operatorname{sgn}\left\{X R_{1}(n, m)\right\} \bullet \min$

$\left\{\left|X R_{1}(n, m)\right|,\left|X R_{2}(n, m)\right|\right\}$

Similarly, if $X I_{1}(n, m)$ and $X I_{2}(n, m)$ are different, then it is taken:

$$
Y_{I}(n, m)=0
$$

If $X I_{1}(n, m)$ and $X I_{2}(n, m)$ are the same, then it is taken: 


$$
\begin{aligned}
& Y_{I}(n, m)=\operatorname{sgn}\left\{X I_{1}(n, m)\right\} \bullet \min \\
& \left\{\left|X I_{1}(n, m)\right|,\left|X I_{2}(n, m)\right|\right\}
\end{aligned}
$$

The signal $Y(n, m)$ for enhanced processing is:

$$
Y(n, m)=Y_{R}(n, m)+j Y_{I}(n, m)
$$

Figure 1 shows the result diagram of the ideal point target enhancement processing. It is shown that if the original data $X_{2}(n, m)$ were obtained by hanning weighted processing, the obtained $X_{1}(n, m)$ effectively reduces the side lobe, but it also increases the width of the main lobe, loss of resolution. By using the method proposed $Y(n, m)$ in this paper, not only the sidelobe is effectively suppressed, but also the main lobe width is exactly the same with the original data, which preserves the high resolution characteriation after good

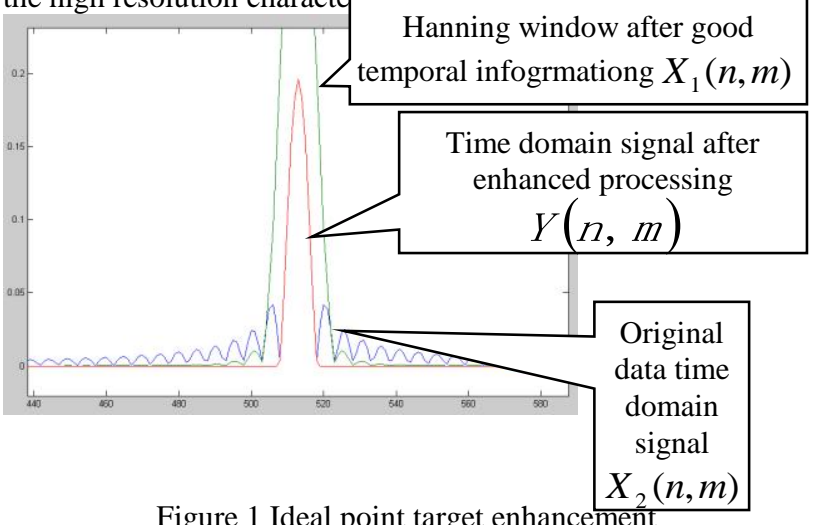

Figure 1 Ideal point target enhancement processing result diagram

\section{IMAGE ENHANCEMENT PROCESSING RESULT}

\subsection{Simulation point target enhancement processing}

The frequency spectrum of the point target LFM signal is a constant, and a constant signal is constructed in the effective frequency spectrum of the frequency domain. After enhanced processing, the index is as follows:

\begin{tabular}{|c|c|c|c|}
\hline & $\begin{array}{c}\text { Original } \\
\text { signal }\end{array}$ & $\begin{array}{c}\text { Post } \\
\text { window } \\
\text { signal }\end{array}$ & $\begin{array}{c}\text { Enhanced post } \\
\text { processing } \\
\text { signal }\end{array}$ \\
\hline $\begin{array}{c}\text { Peak } \\
\text { sidelobe } \\
\text { ratio }\end{array}$ & -13.2467 & -37 & -37 \\
\hline $\begin{array}{c}\text { Main } \\
\text { lobe } \\
\text { width }\end{array}$ & 4.515625 & 7.328125 & 4.515625 \\
\hline
\end{tabular}

Table1 Resolution improvement index of enhanced processing algorithm

It can be seen that compared with the traditional imaging processing method, the above enhancement processing method can get the peak sidelobe ratio of $-37 \mathrm{~dB}$, and the resolution can be increased by $62.28 \%(7.328125 / 4.515625-1)$ times.

\subsection{Airborne real data enhancement processing}

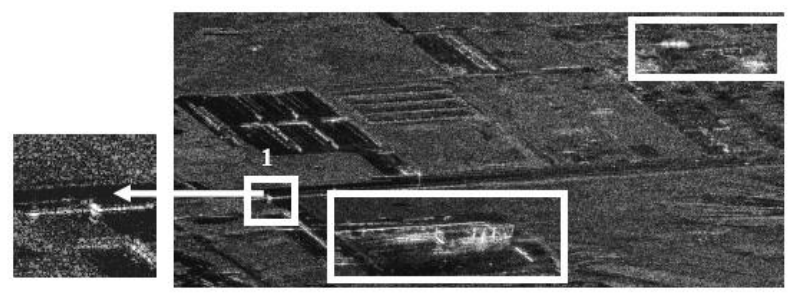

Figure 2 Airborne SAR original image

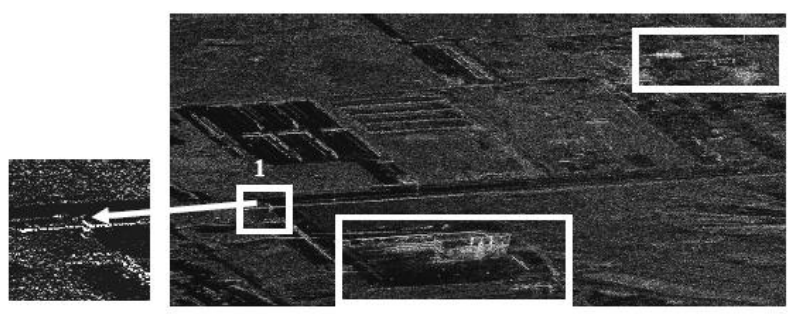

Figure 3 Image after enhanced processing of airborne SAR

By comparing and analyzing figures 2 and 3, we can see that after enhanced processing, the image is clearer than that before processing, especially the sidelobe of the logo area is significantly reduced, and the corner reflection phenomenon is obviously weakened. The 1 blurred line of the two lines is distinctly separated by processing.

\section{CONCLUSION}

The resolution of the SAR image directly reflects the main information obtained by the radar, and is the main technical indicator of the synthetic aperture radar (SAR) satellite. The enhancement of resolution can enhance the details and texture features of the target, and it is of great significance to target recognition, change detection and target state evaluation. Because of the high cost of high resolution SAR images on the star, increasing resolution through ground processing has attracted more attention from the SAR academia. Resolution enhancement processing is also a technical measure that is often used in the foreign radar satellite system. Through the study of this paper proves that: in keeping the same under the condition of the sidelobe level $(30 \mathrm{~dB})$, using resolution enhancement methods of SAR image processing technology to obtain the general resolution is about $50 \%$.

\section{REFERENCES}

Xue Guoyi.Research of Montion Compensation in Airborne UWB SAR with High Resolution. Doctoral thesis of National University of Defense Technology, 2008 : 36-38.

Yang Dan.Motion Compensation for airborne SAR Interferometry. Master's thesis of Xi'an Electronic and Science University, 2011:46-78.

Richard Baraniuk and Philippe Steeghs. Compressive radar imaging. IEEE Radar Conference, Waltham, Massachusetts, April 2007.

M.A.Herman and T.Strohmer. High-Resolution Radar via Compressed Sensing. IEEE Transactions on Signal Processing Vol. 57,No.6, pp.2275-2284,June 2009. 